\title{
Design of cloaked Yagi-Uda antennas
}

\author{
Alessio Monti ${ }^{1, *}$, Jason Soric ${ }^{2}$, Andrea Alù ${ }^{2}$, Alessandro Toscano ${ }^{3}$, and Filiberto Bilotti ${ }^{3}$ \\ 1 Engineering Department, "Niccolò Cusano" University, Via Don Carlo Gnocchi 3, 00166 Rome, Italy \\ 2 Department of Electrical and Computer Engineering, The University of Texas at Austin, Austin, TX 78712, USA \\ 3 Department of Engineering, "Roma Tre" University, Via Vito Volterra 62, 00146 Rome, Italy
}

Received 7 September 2016 / Accepted 24 October 2016

\begin{abstract}
Exploiting the appealing features of the mantle cloaking approach, we propose a general procedure that allows designing Yagi-Uda antennas with low scattering profile. The proposed radiators are able to operate efficiently within a given frequency band, while being electromagnetically undetectable in another desired frequency range. The general design procedure of such cloaked antennas is first presented, considering that both antenna and cloak requirements must be simultaneously fulfilled. Then, we prove that a cloaked Yagi-Uda antenna can be installed in close proximity to other radiators without affecting their performance. Due to these unprecedented characteristics, the proposed radiators may have a strong impact in the design of compact overcrowded platforms for both terrestrial and satellite applications.
\end{abstract}

Key words: Electromagnetic cloaking, Yagi-Uda antennas, Metasurfaces.

\section{Introduction}

In the last decade, the development of cloaking technologies [1-9] has enabled an unprecedented control of the electromagnetic scattering proprieties of an illuminated object of choice. Differently from well-established radar cross section reduction (RCSR) techniques [10], in fact, cloaking allows reducing the overall scattering cross section (SCS) of an object, making it significantly less detectable to an external observer or to a sensor within the desired frequency range. Among the various cloaking techniques proposed so far, mantle cloaking $[5,6]$ is particularly promising for antenna applications at microwave frequencies, due to its straightforward implementation [11] and the availability of accurate analytical models [12, 13] resulting in quick and effective design procedures. Moreover, as it happens in any scattering cancellation based approach to cloaking, the concealed object is not electromagnetically isolated and, thus, the mantle cloaking can be successfully applied to different antenna scenarios, with remarkable advantages for both transmitting [14-19] and receiving [20] antennas.

In this work, we exploit the above-mentioned peculiarities of mantle cloaking to propose an original antenna configuration that is specifically designed to work in a given frequency range, while being electromagnetically undetectable in another one. This layout may play an important role in the

*e-mail: alessio.monti@unicusano.it design of modern communication platforms hosting an everincreasing number of antennas operating for different services at different frequencies. The possibility to use cloaked antenna systems that do not affect the performance of the other radiators placed nearby, in fact, can result in a dramatic reduction of weight and space occupancy of complex electromagnetic platforms, such as civil and military vehicles, as well as satellite payloads.

We focus our attention on arguably the most popular and widely used antenna, i.e., the Yagi-Uda radiator, and show how it is possible to design a version of such an antenna operating at UHF frequencies, which presents very low scattering within a wide range of the $\mathrm{S}$ band. The proposed design guidelines take into account numerous issues arising in a realistic design, such as the complex scattering effects due to finite-length metallic cylinders of the Yagi-Uda antenna and the needed trade-off between cloaking and antenna performance.

The design procedure presented in this work can be applied to any frequency range of interest and considered as a nontrivial generalization of our previous results on the cloaking of finite-length metallic cylinders $[11,13]$ and dipole antennas [14, 19]. Compared to these earlier results, in fact, the presence of two or more parasitic elements acting as loads of the driven dipole introduces significant complications in the achievement of a final working design. Moreover, the design proposed here exhibits a dramatically broader operation band compared to the idealized cases discussed in previous works (see, e.g., [14]). 


\section{Design guidelines}

In this section, we summarize the guidelines for the design of a cloaked Yagi-Uda antenna. Hereinafter, we denote with $\mathrm{BW}_{\mathrm{a}}$ the operation frequency range $\left(f_{1 a}-f_{2 a}\right)$ of the Yagi-Uda antenna and with $\mathrm{BW}_{\mathrm{b}}$ the frequency range $\left(f_{1 b}-f_{2 b}\right)$ in which we desire to make it low-scattering. In our design, we choose the frequency range $896-970 \mathrm{MHz}$ [21] as $\mathrm{BW}_{\mathrm{a}}$, and the range 2.6-3.9 $\mathrm{GHz}$ as $\mathrm{BW}_{\mathrm{b}}$. However, we emphasize here that the same design procedure is effective for any two distinct frequency ranges of interest radiofrequencies and microwaves. As it will be clear later, our choice is motivated by the fact that the proposed frequency ranges lead to a case for which the final design is particularly challenging.

Step 1: The first design step consists in the synthesis of a regular Yagi-Uda antenna. In our case, we have considered a 3 -element radiator whose geometry is reported in Figure 1a. Following the design recommendations in [22], we have fixed the following initial geometrical parameters: $L_{r}=0.52 \lambda_{0 a}$, $L_{c}=0.47 \lambda_{0 a}, L_{d}=0.43 \lambda_{0 a}, d_{c d}=0.35 \lambda_{0 a}$ and $d_{c r}=0.25 \lambda_{0 a}$, where $\lambda_{0 a}$ is the wavelength at the center frequency of $\mathrm{BW}_{\mathrm{a}}$. It is important to note that the choice of the radii of the three cylinders $r_{r}, r_{c}$ and $r_{d}$ requires a special attention, since it represents the first trade-off between the Yagi-Uda performance, in particular its bandwidth, and the cloak performance. In fact, a single-layer scattering cancellation device is effective as long as the radius of the hidden object is electrically small compared to the cloaking wavelength (i.e., $r_{r}, r_{c}, r_{d}<<\lambda_{0 b}$, where $\lambda_{0 b}$ is the wavelength at the center frequency of $\mathrm{BW}_{\mathrm{b}}$ ) [23]. In our design, for which $\lambda_{0 a}=3.5 \lambda_{0 b}$, we can safely fix all the radii equal to $\lambda_{0 a} / 107=\lambda_{0 b} / 31$. As it will be clear later, this choice allows achieving both the desired antenna bandwidth $\mathrm{BW}_{\mathrm{a}}$ and significant cloaking performances within $\mathrm{BW}_{\mathrm{b}}$. In the uncommon scenarios in which non-electrically small radii are required, it would be possible to deal with the enhanced scattering by employing multilayered cloaks $[24,25]$.

After optimization of the geometrical parameters conducted through a full-wave simulator [26], we have achieved the final design reported in Table 1 (second column) and characterized by the electrical and radiative characteristics shown in Figures 2 and 3, respectively. As desired, the antenna is matched in $\mathrm{BW}_{\mathrm{a}}$ and exhibits a front-to-back ratio (FBR) approximately equal to $11.5 \mathrm{~dB}$ at its center frequency.

Step 2: In order to properly take into account the effects of the cloaking device on the Yagi-Uda characteristics, the second step of the design consists in covering each of the three elements composing the antenna with proper mantle cloaks, as shown in Figure 1b. As is now well known, a mantle cloak is made of a properly patterned metallic surface (i.e., a metasurface) supported by a dielectric layer and exhibiting a desired value of intrinsic surface reactance $X_{\mathrm{s}}(\Omega / \mathrm{sq})$ [12, 13]. Since the cloaking of a metallic cylinder requires a capacitive metasurface [7], the generic cloaks shown in Figure $1 \mathrm{~b}$ are made of thin horizontal metallic strips.

In this step, the cloaks' radii and the choice of the supporting dielectric represent critical elements of the design. These parameters, in fact, affect both the Yagi-Uda antenna (a)

(b)
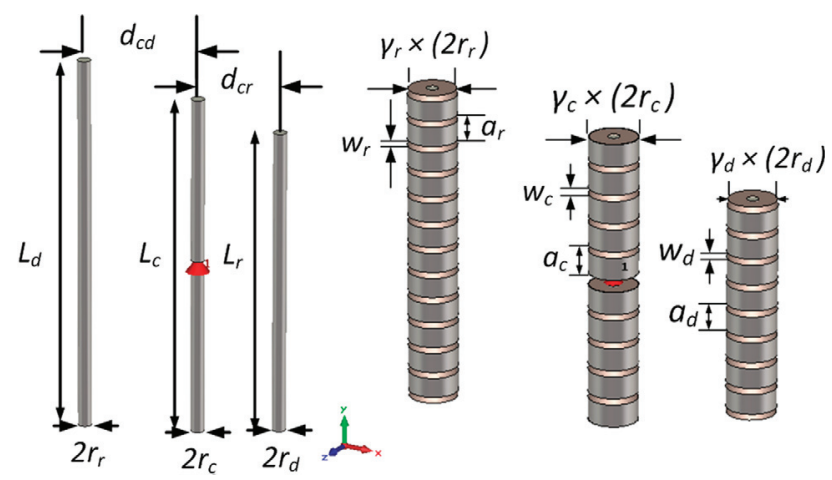

Figure 1. (a) Geometry of a regular Yagi-Uda antenna. (b) Geometry of a cloaked Yagi-Uda antenna in which the three metallic rods are surrounded by mantle cloaks. $\gamma$ is the ratio between the cover and the cylinder radius.

Table 1. Geometrical parameters of the uncloaked and of the cloaked Yagi-Uda antenna.

\begin{tabular}{lcc}
\hline Geometrical parameter & Uncloaked & Cloaked \\
\hline$L_{r}$ & $\lambda_{0 a} / 2.13$ & $\lambda_{0 a} / 2.33$ \\
$L_{c}$ & $\lambda_{0 a} / 2.56$ & $\lambda_{0 a} / 3.18$ \\
$L_{d}$ & $\lambda_{0 a} / 3.12$ & $\lambda_{0 a} / 4.71$ \\
$d_{c r}$ & $\lambda_{0 a} / 3.35$ & $\lambda_{0 a} / 3.77$ \\
$d_{c d}$ & $\lambda_{0 a} / 4.45$ & $\lambda_{0 a} / 7.43$ \\
$r_{r}$ & $\lambda_{0 a} / 109$ & $\lambda_{0 a} / 109$ \\
$r_{c}$ & $\lambda_{0 a} / 109$ & $\lambda_{0 a} / 85$ \\
$r_{d}$ & $\lambda_{0 a} / 109$ & $\lambda_{0 a} / 109$ \\
\hline
\end{tabular}

and the cloak performance. According to the results reported in [11], we can state, as a general rule, that the use of a dielectric with high permittivity improves the cloaking performance in terms of overall scattering reduction and lowprofile, but it also leads to a reduction of cloaking bandwidth and input resistance of the Yagi-Uda element. The use of large cloaks radii, instead, improves the cloaking bandwidth but reduces the input resistance of the antenna.

A good trade-off among these different requirements can be obtained by using a substrate made of Teflon, a low-loss and easily machinable material with moderate permittivity $\varepsilon_{r}=2.1$ and low loss-factor $\tan \delta=0.0002$, and fixing $\gamma_{r}, \gamma_{c}$ and $\gamma_{d}$ equal to 3 . The application of the generic cloaks around the Yagi-Uda elements introduces a frequency redshift of the antenna operation band, which is mainly due to the dielectric used to support the metasurfaces placed around the cylinders.

Step 3: This step is focused on the restoration of the proper operation of the Yagi-Uda deteriorated by the application of the cloaks within $\mathrm{BW}_{\mathrm{a}}$. A first solution to achieve this goal is to scale all the antenna dimensions by a proper geometrical factor. Despite its simplicity, this solution is the best one in many regards. However, there are some scenarios, as the one at hand, in which antenna scaling is not a viable solution. In fact, the finite length of the cylinders composing the Yagi-Uda array introduces complex resonant effects [27, 28] whose scattering cannot be straightforwardly compensated. 


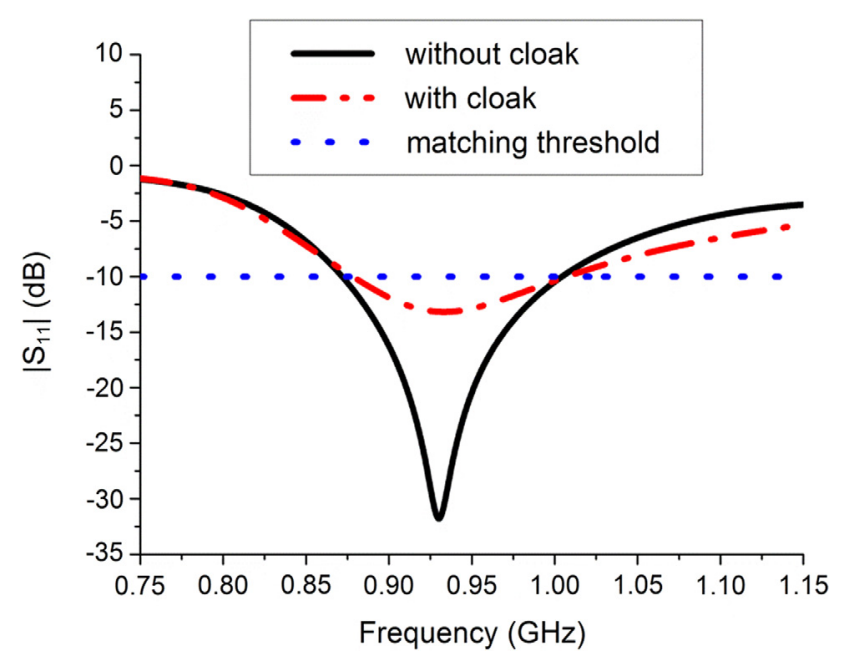

Figure 2. Magnitude of the reflection coefficient of the bare (continuous line) and of the cloaked (dash-dotted line) Yagi-Uda antenna. The dotted line represents the commonly used $-10 \mathrm{~dB}$ matching threshold.

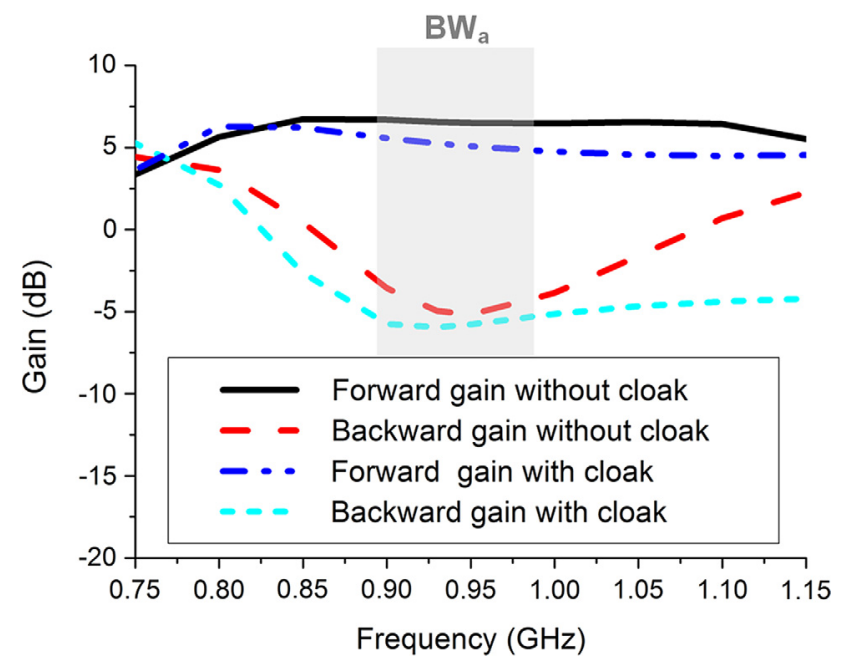

Figure 3. Forward and backward gain of the bare Yagi-Uda antenna compared to the one achieved with a cloaked antenna.

These resonances can be avoided if the length of the cloaked parasitic elements of the Yagi-Uda do not excessively approach multiples of half wavelength in the metasurface dielectric of the cloaking band $\mathrm{BW}_{\mathrm{b}}$. For our design, in particular, the critical element is the length of the director, which needs to be less than $\lambda_{2 b} / \sqrt{\varepsilon_{r}}$, being $\lambda_{2 b}$ the lower wavelength of the range $\mathrm{BW}_{\mathrm{b}}$. A simple antenna scaling would not allow satisfying this condition, necessitating further optimization.

In particular, after having restored the antenna resonance by reducing the length of the three cylinders, it is necessary to optimize the length of the parasitic elements $\left(L_{d}\right.$ and $\left.L_{r}\right)$, their radii $\left(r_{d}\right.$ and $\left.r_{r}\right)$, and their separations from the driven dipole $\left(d_{c d}\right.$ and $\left.d_{c r}\right)$, taking into account the previously defined bounds. The main issue caused by the cloaks is the reduction of the antenna input resistance, which is difficult to match to the required $50 \Omega$ while keeping the original antenna FBR. The numerical optimization leads to the geometrical parameters reported in the third column of Table 1. The relative antenna performance is shown in Figures 2 and 3. As it can be appreciated, the optimized cloaked antenna is matched over $\mathrm{BW}_{\mathrm{a}}$ and exhibits a FBR equal to $11.2 \mathrm{~dB}$.

It is worth noticing that, though the FBR is almost equal in the two cases, the forward gain of the cloaked Yagi-Uda antenna is slightly smaller compared to the one achieved in the uncloaked case. We point out that if we allow an external device to achieve impedance matching at $50 \Omega$ and, so, we can neglect the value of the input resistance in the optimization of the cloaked Yagi-Uda antenna, a higher forward gain may be achieved, even in the cloaked case. We only mention this possibility for the sake of completeness without entering into this discussion further, since the aim of this work is to propose a self-matched design.

\section{Cloak design}

After having optimized the cloaked Yagi-Uda antenna, in this section we focus our attention to the design of the cloaks applied to the three elements of the Yagi-Uda. As already mentioned in the previous section and shown in Figure 1b, these capacitive cloaks are simply made of horizontal metallic strips engraved on a dielectric support. The periodicity $a$ and the separation $w$ between strips strongly affect the surface reactance exhibited by the cloaks at $\lambda_{0 b}$, but do not affect the performance of the Yagi-Uda in $\mathrm{BW}_{\mathrm{a}}$. The surface reactance formula used to design the cloaks is [12]:

$$
Z_{\mathrm{s}}=-j \frac{\pi}{2 \omega \varepsilon_{0} \mathrm{a} \ln \left[\csc \left(\frac{\pi w}{2 a}\right)\right]}
$$

where $\omega$ is the desired cloaking frequency (e.g., in our case, it corresponds to the central frequency of $\mathrm{BW}_{\mathrm{b}}$ ). Please note that, though derived in a planar configuration, the accuracy of this formula for cylindrical geometry has been confirmed in several previous publications $[10,11]$.

The required surface reactances to cloak the three finitelength cylinders composing the Yagi-Uda antenna at the central frequency of $\mathrm{BW}_{\mathrm{b}}$ can be computed following the procedure outlined in [13]. Once these values are known, by using (Eq. (1)) it is possible to quickly design three horizontal-strip metasurfaces. The geometrical parameters of the final cloaks, made of $N_{r}=12$ and $N_{c}=N_{d}=8$ rows, respectively, are reported in Table 2, whereas in Figure 4 we show the dispersive behavior of the designed metasurfaces, retrieved using a simple full-wave procedure based on a transmission-line model [13]. Due to its sub-wavelength pattern, the designed cloaks can replace the ideal ones used during the Yagi-Uda optimization without affecting the antenna performance. We have carefully verified this expected property through full-wave simulations.

In Figure 5, we show the total scattering cross section SCS (i.e., the radar cross section integrated all around the solid angle) of both the uncloaked and cloaked Yagi-Uda antenna when it is illuminated by an external plane-wave whose electric field is parallel to the axis of the cylinders. We have achieved a 14.6 $\mathrm{dB}$ reduction of the total SCS around the central frequency of $\mathrm{BW}_{\mathrm{b}}$ with $\mathrm{a}-10 \mathrm{~dB}$ bandwidth of about $400 \mathrm{MHz}$. 
Table 2. Geometrical parameters of the three metasurfaces used to cloak the designed Yagi-Uda antenna.

\begin{tabular}{lr}
\hline Geometrical parameter & Value \\
\hline$a_{r}$ & $\lambda_{0 b} / 7.9$ \\
$a_{c}$ & $\lambda_{0 b} / 8.0$ \\
$a_{d}$ & $\lambda_{0 b} / 11.1$ \\
$w_{r}$ & $\lambda_{0 b} / 73.8$ \\
$w_{c}$ & $\lambda_{0 b} / 185.5$ \\
$w_{d}$ & $\lambda_{0 b} / 307.5$ \\
\hline
\end{tabular}

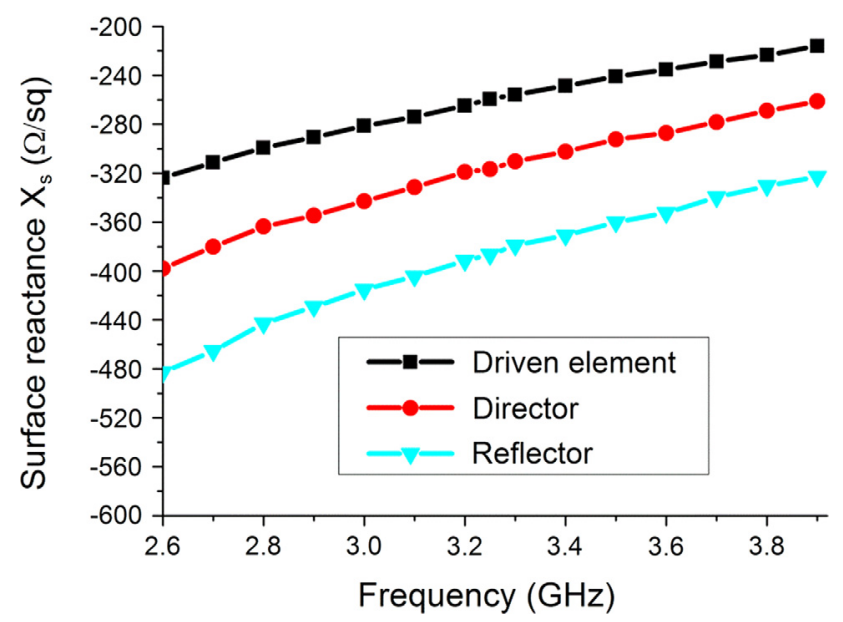

Figure 4. Retrieved surface reactance of the three designed horizontal strip metasurfaces as a function of frequency.

These results confirm that the designed cloaks have an operative bandwidth dramatically broader compared to the ones obtained in earlier papers [14-17]. We emphasize here that the improvement of the cloak performance has been obtained through the accurate choice of both cloak radii and dielectric support of the metasurfaces. As already discussed, the use of cloaks with smaller radii would result in an increase of the antenna input resistance but it is inevitably related to a reduction of the cloaking bandwidth.

We can conclude this section by stressing the fact that, thanks to the application of the designed cloaks, the proposed Yagi-Uda antenna is significantly less visible to radiowaves in $\mathrm{BW}_{\mathrm{b}}$ while it keeps the ability to properly operate as an efficient radiator in $\mathrm{BW}_{\mathrm{a}}$.

\section{Use of cloaked Yagi-Uda antennas in realistic scenarios}

In this section, we check the capability of the designed cloaked Yagi-Uda antenna to weakly affect other radiators placed in its close vicinity. As a first example, we consider the scenario depicted in the inset of Figure 6, in which a Yagi-Uda radiator obstructs an $11 \mathrm{~dB}$ WR-284 horn antenna whose mono-modal band coincides with the range $\mathrm{BW}_{\mathrm{b}}$ adopted in this paper [21]. The distance between the two antennas is equal to $\lambda_{0 b} / 2$. In Figure 6 , we show the comparison between the magnitude of the reflection coefficient of the horn

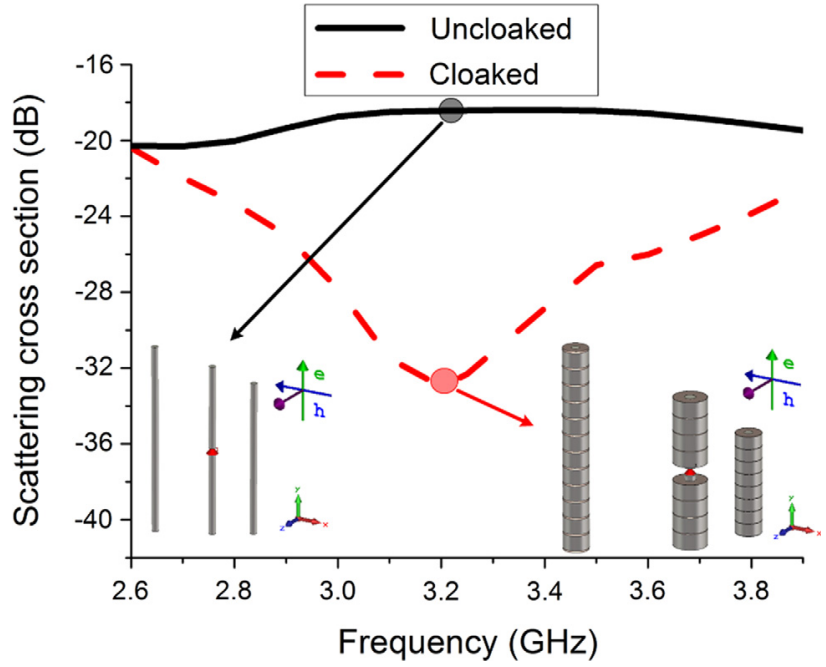

Figure 5. Total SCS of the Yagi-Uda antenna in the uncloaked (continuous line) and cloaked (dashed line) cases under a TM planewave illumination. Please note that the antenna scattering is reduced within the whole $\mathrm{BW}_{\mathrm{b}}$ frequency range.

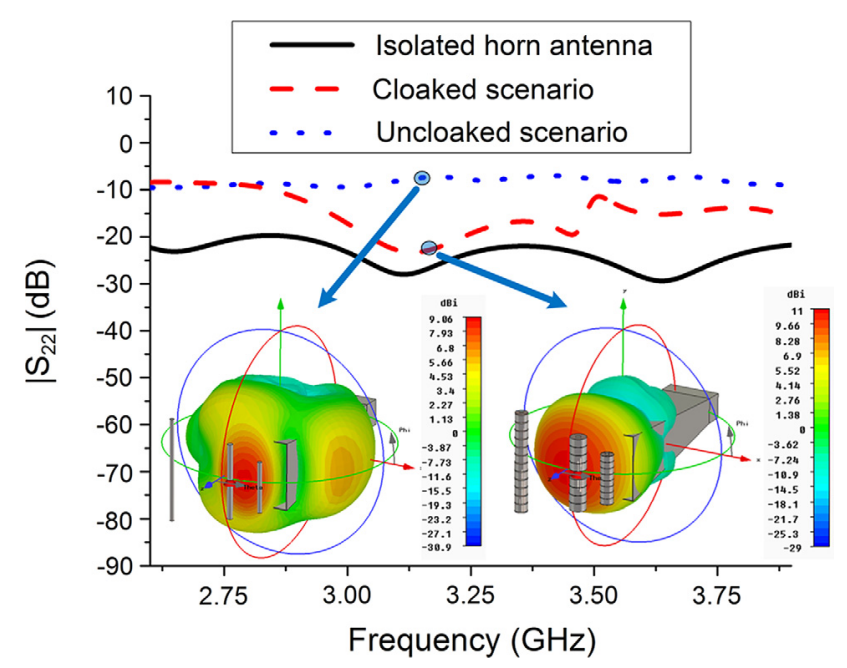

Figure 6. Magnitude of the reflection coefficient of the horn antenna in the isolated (continuous line), uncloaked (dotted line) and cloaked (dashed line) cases.

antenna in three cases: the isolated case (i.e., unobstructed horn antenna), the uncloaked case (i.e., horn antenna obstructed by an uncloaked Yagi-Uda radiator) and the cloaked case (i.e., horn antenna obstructed by a cloaked Yagi-Uda radiator designed through the proposed procedure). As expected, the horn impedance matching in the uncloaked case is rather poor, due to the presence of the Yagi-Uda antenna that introduces a significant perturbation in the horn near field. Instead, in the cloaked case the horn impedance matching is dramatically improved, especially within the cloaking bandwidth in which the horn does not experience the presence of the Yagi-Uda despite its very close proximity. In the inset of the same picture, it is also shown the comparison between the horn $3 \mathrm{D}$ radiation pattern in the uncloaked and 
(a)

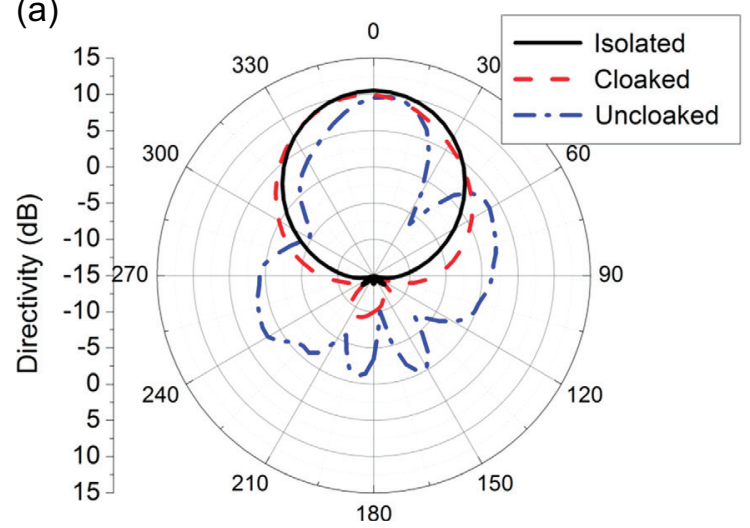

(b)

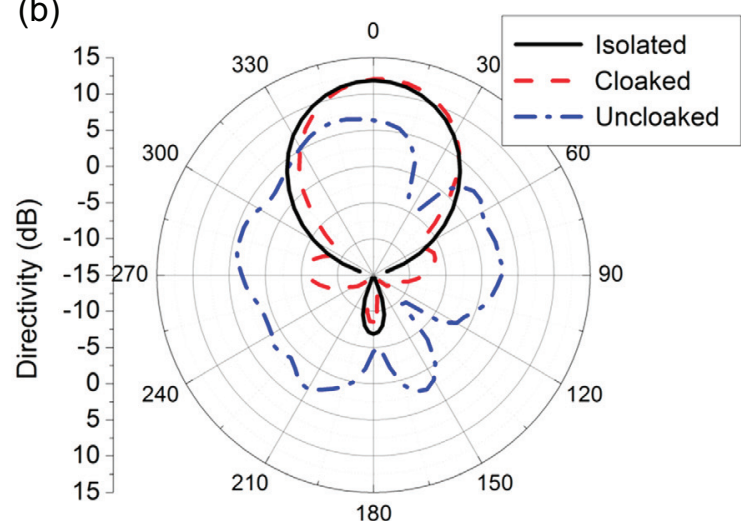

Figure 7. Radiation pattern of the horn antenna on the horizontal plane at (a) $3.1 \mathrm{GHz}$ and (b) $3.6 \mathrm{GHz}$ in the isolated (continuous line), uncloaked (dash-dotted line) and cloaked (dashed line) cases.

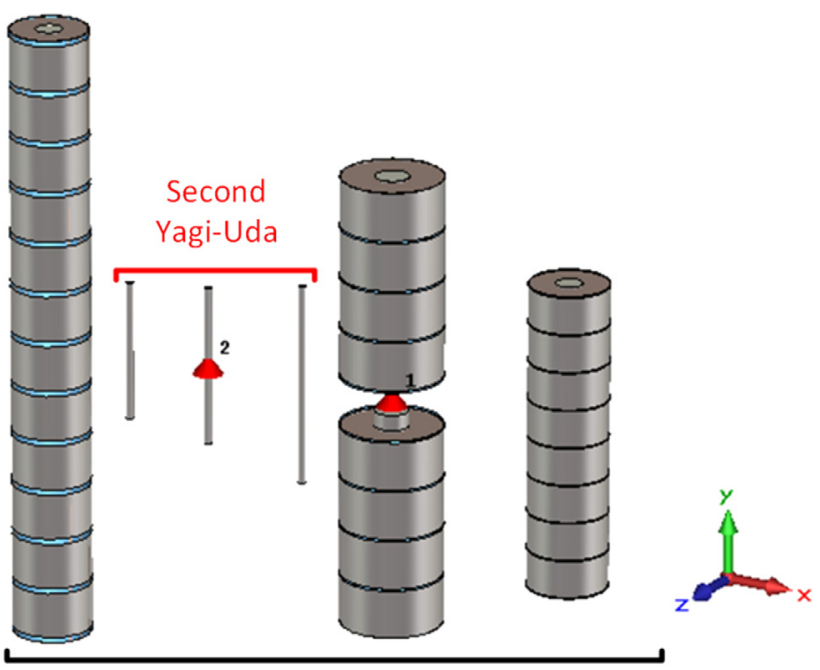

First Yagi-Uda

Figure 8. Antenna scenario in which a Yagi-Uda antenna operating at $3.2 \mathrm{GHz}$ ("second Yagi-Uda") is placed between the director and the driven element of the cloaked Yagi-Uda antenna ("first YagiUda" operating at $0.93 \mathrm{GHz}$ ). Thanks to the cloaks, this system acts as a high directivity and extremely compact dual-band radiator with opposite main lobe directions.

cloaked cases at the central frequency of $\mathrm{BW}_{\mathrm{b}}$. While a dramatic deterioration of the horn radiation pattern can be observed in the uncloaked case, the original horn pattern is totally restored in the cloaked one, confirming the effectiveness of the proposed device.

In Figures 7, we compare the horn radiation pattern on the horizontal plane at the frequencies $3.1 \mathrm{GHz}$ and $3.6 \mathrm{GHz}$, respectively. It is evident that the restoration of the original horn radiative characteristics is obtained in a wide range of frequencies and not only at the cloaking frequency. Outside this range, the horn pattern is progressively deteriorated due to the reduction of the cloak performances (see Figure 5). Finally, we underline that the presence of a thin horizontal metallic structure supporting the elements of the Yagi-Uda would not affect the cloaking performances due to its rather moderate

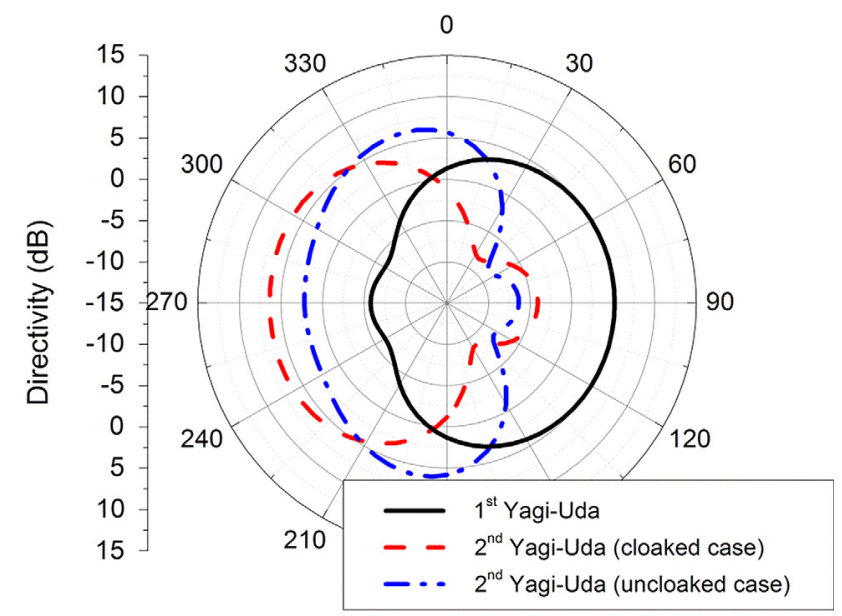

Figure 9. Directivity on the $x-z$ plane of the two Yagi-Uda antennas system shown in Figure 8. The directivity of the second Yagi-Uda is shown both in the uncloaked and in the cloaked scenario.

scattering for a vertical incidence polarization (as the one emerging from the horn antenna aperture).

As further evidence of the advantages provided by the cloaked Yagi-Uda antennas, we have explored the challenging scenario sketched in Figure 8. In this case, a Yagi-Uda antenna optimized to work in a sub-range of the cloaking band $\mathrm{BW}_{\mathrm{b}}$ (denoted with the term "second Yagi-Uda") is placed between the driven element and the reflector of the cloaked Yagi-Uda antenna that operates at $\mathrm{BW}_{\mathrm{a}}$ (hereinafter "first Yagi-Uda"). The second Yagi-Uda is intentionally oriented to radiate in the opposite direction compared to the first one. As expected and reported in Figure 9, in the uncloaked scenario the presence of the first Yagi-Uda dramatically deteriorates the performances of the second one. In the cloaked scenario, instead, the second Yagi-Uda antenna correctly radiates in an opposite direction compared to the first one and operates as the first Yagi-Uda were not present (Figure 9). Moreover, in the same picture, it is possible to appreciate that the first Yagi-Uda is not affected by the presence of the second one, due its reduced electrical dimensions in $\mathrm{BW}_{\mathrm{a}}$. Therefore, the 
(a)

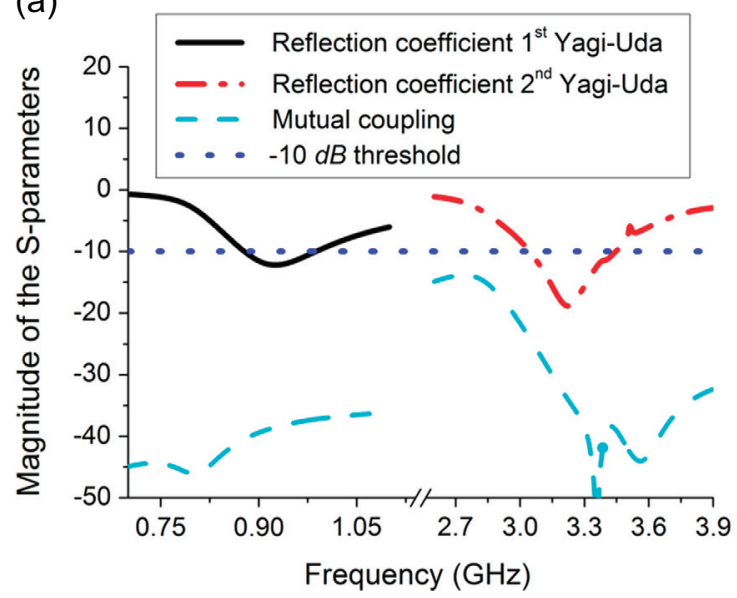

(b)

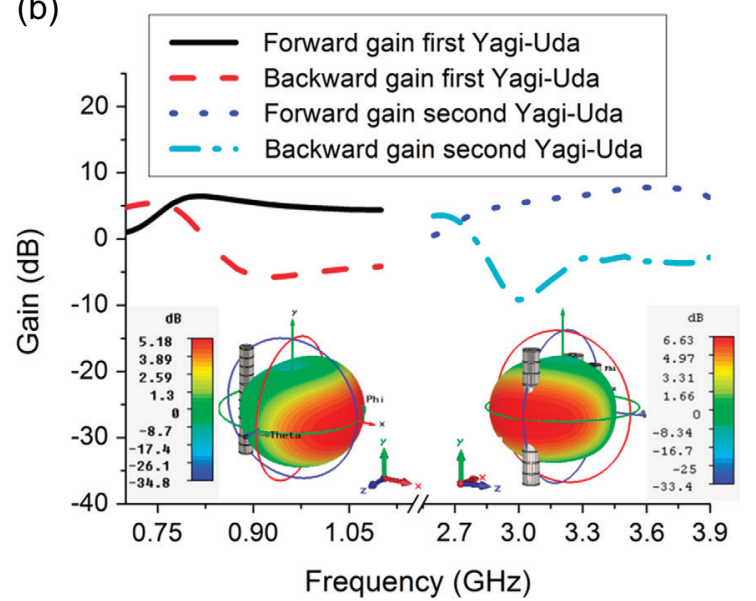

Figure 10. (a) Magnitude of the S-parameters of the two-Yagi Uda antenna system depicted in Figure 8 and relative (b) forward and backward gain. In the insets, it is possible to appreciate the 3D radiation pattern of the first Yagi-Uda at $0.93 \mathrm{GHz}$ and of the second one at $3.2 \mathrm{GHz}$, respectively. Please note that, as desired, the two antennas radiate in opposite direction and do not affect each other in terms of both electrical and radiative characteristics.

antenna system shown in Figure 8, acts as an innovative high-directivity and extremely compact dual-band radiator with opposite main lobe directions at its two resonance frequencies.

In Figure 10, we show the S-parameters and the forward and backward gain of the two Yagi-Uda antennas as a function of frequency. The inset of Figure $10 \mathrm{~b}$ shows the 3D radiation pattern at the central frequency of $\mathrm{BW}_{\mathrm{a}}$ and $\mathrm{BW}_{\mathrm{b}}$, respectively. As it can be appreciated in the figure, thanks to the cloaks each Yagi-Uda antenna properly operates over its own bandwidth without affecting the performance of the other antenna. Notably, even the mutual coupling between the two antennas is extremely low in both operational bandwidths despite their extreme proximity.

\section{Conclusions}

In this contribution, we have investigated the design of cloaked Yagi-Uda antennas by exploiting the mantle cloaking technique. In particular, we have outlined a general procedure that allows designing a cloaked Yagi-Uda antenna properly operating in its bandwidth and featuring drastically reduced scattering in another desired frequency range. These innovative antenna systems represent a non-trivial generalization of cloaked dipoles proposed in earlier works, because their design is complicated by the presence of parasitic elements and more complex scattering effects that should to be taken into proper account in both the antenna and the cloaks design. The possibility to design cloaked Yagi-Uda antennas, as discussed in this paper, further increases the potentialities of electromagnetic cloaking in the design of compact communication platforms in which the different antennas can be placed in strong proximity without deteriorating their performances. The cloaked Yagi-Uda antenna has been checked both for an ideal electromagnetic excitation (e.g., transverse-magnetic (TM) plane wave) and in realistic antenna scenarios. Finally, we have shown that the use of cloaked Yagi-Uda antennas allows designing extremely compact dual-band Yagi-Uda radiators with opposite main lobe directions.

\section{Fundings information}

J.S. and A.A. were supported by the Air Force Office of Scientific Research and the Defense Threat Reduction Agency.

\section{References}

1. R. Fleury, F. Monticone, A. Alù, Phys. Rev. Applied 4 (2015) 037001.

2. J.B. Pendry, D. Schurig, D.R. Smith, Science 312 (2006) 1780.

3. P. Alitalo, O. Luukkonen, L. Jylha, J. Venermo, S.A. Tretyakov, IEEE Trans. Antennas Propag. 56 (2008) 416.

4. P.-S. Kildal, A.A. Kishk, A. Tengs, IEEE Trans. Antennas Propag. 44 (1996) 1509.

5. A. Alù, N. Engheta, Phys. Rev. E 72 (2005) 016623.

6. A. Alù, Phys. Rev. B 80 (2009) 24115.

7. P.Y. Chen, A. Alù, Phys. Rev. B 84 (2011) 205110.

8. M. Selvanayagam, G.V. Eleftheriades, IEEE Antennas Wirel. Propag. Lett. 11 (2012) 1226.

9. F. Monticone, A. Alù, Optica 3 (2016) 718.

10. E. Knott, M.T. Tuley, J.F. Shaeffer, Radar cross section second edition, Artech House Publishers, Norwood, MA, 1993.

11. J.C. Soric, P.Y. Chen, A. Kerkhoff, D. Rainwater, K. Melin, A. Alù, New J. Phys. 15 (2013) 033037.

12. Y.R. Padooru, A.B. Yakovlev, P. Chen, A. Alù, J. Appl. Phys. 112 (2012) 034907.

13. A. Monti, J. Soric, A. Alù, A. Toscano, F. Bilotti, IEEE Trans. Antennas Propag. 63 (2015) 1775.

14. A. Monti, J. Soric, A. Alù, F. Bilotti, A. Toscano, L. Vegni, IEEE Antennas Wirel. Propag. Lett. 11 (2012) 1414.

15. M.H. Bernety, A. Yakovlev, IEEE Trans. Antennas Propag. 63 (2015) 1554 
16. Z.H. Jiang, P.E. Sieber, L. Kang, D.H. Werner, Adv. Funct. Mater. 25 (2015) 4708.

17. M.H. Bernety, A. Yakovlev, J. Appl. Phys. 119 (2016) 014904.

18. T.V. Teperik, A. de Lustrac, AIP Advances 5 (2015) 127225.

19. A. Monti, J. Soric, M. Barbuto, D. Ramaccia, S. Vellucci, F. Trotta, A. Alù, A. Toscano, F. Bilotti, Appl. Phys. Lett. 108 (2016) 113502.

20. J.C. Soric, R. Fluery, A. Monti, A. Toscano, F. Bilotti, A. Alù, IEEE Trans. Antennas Propag. 62 (2014) 4220.

21. Pasternack ${ }^{\circledR}$, John Wiley \& Sons, Hoboken, NJ, www.pasternack.com/directional-antennas-category.aspx.

22. C.A. Balanis, Antenna theory: analysis and design, John Wiley \& Sons, 2005, pp. 577-600.
23. C.F. Bohren, D.R. Huffman, Absorption and scattering of light by small particles, Wiley-Interscience, New York, 1998.

24. A. Monti, L. Tenuti, G. Oliveri, A. Alù, A. Massa, A. Toscano, F. Bilotti, Design of multi-layer mantle cloaks, Proceeding of the 8th International Congress on Advanced Electromagnetic Materials in Microwaves and Optics, Copenhagen, Denmark, 25-28, August 2014, pp. 214-216

25. J. Soric, A. Monti, A. Toscano, F. Bilotti, A. Alù, IEEE Trans. Antennas Propag. 63 (2015) 3235.

26. CST Microwave Studio 2014, www.cst.com

27. D. Brill, G.C. Gaunard, H. Huang, Proceedings of SPIE 2485 (1995) 187.

28. J.H. Van Vleck, F. Bloch, M. Hamermesh, J. Appl. Phys. 18 (1947) 274.

Cite this article as: Monti A, Soric J, Alù A, Toscano A \& Bilotti F: Design of cloaked Yagi-Uda antennas. EPJ Appl. Metamat. 2016, 3, 10 . 